\title{
Die Sichtweise von Schülerinnen und Schülern auf eine primärpräventive Maßnahme eines ehemaligen Rechtsextremen: Befunde einer Evaluationsstudie und Empfehlungen für Bildungsakteure
}

\author{
Antje Gansewig (D) Maria Walsh
}

Eingegangen: 1. November 2020 / Überarbeitet: 13. März 2021 / Angenommen: 16. März 2021 / Online publiziert: 4. Mai 2021

(C) Der/die Autor(en) 2021

Zusammenfassung Schulische Veranstaltungen von und mit ehemaligen extrem Rechten finden in Deutschland mittlerweile seit zwei Jahrzehnten statt. Hiermit wird vorrangig die Zielsetzung verfolgt, Radikalisierung und Extremismus vorzubeugen. Das gelinge, so die Grundannahme, durch den biografischen Erfahrungshintergrund der Aussteiger/innen effektiver im Vergleich zu anderen Interventionen. Bemerkenswerterweise ist dieses spezifische Format der schulischen Präventionsund Bildungsarbeit bislang nur marginal wissenschaftlich untersucht worden. Bisher existierten kaum empirisch fundierte Erkenntnisse über die (nicht-)intendierten Effekte derartiger Maßnahmen auf die Schüler/innen sowie deren Sichtweise. Vor diesem Hintergrund erachteten die Autorinnen die Durchführung eines umfassenden Forschungsprojekts zum schulischen Einsatz von früheren Extremist/innen, bei dem das Hauptaugenmerk auf den Schüler/innen liegt, als Erfordernis. Neben einer Bestandserhebung zu entsprechenden Tätigkeiten wurde eine exemplarische primärpräventive Schulmaßnahme eines ehemaligen Rechtsextremen im Rahmen einer Wirkungs- und Prozessevaluation mit randomisiertem Kontrollgruppendesign an 13 öffentlichen Gemeinschaftsschulen in 50 Klassen untersucht. Der vorliegende Beitrag stellt relevante Teilergebnisse dieser Evaluationsstudie vor und diskutiert sie. Im Fokus stehen hierbei die schriftlichen Schüler/innenbefragungen der Prozessevaluation zur Intervention und zum Referenten. Die Ergebnisse der direkt nach der Maßnahme $(n=490)$ und durchschnittlich fünfeinhalb Monate danach $(n=448)$ erhobenen Daten, weisen auf eine heterogene Schüler/innenperspektive und Beson-

\footnotetext{
A. Gansewig ( $\square)$

Fakultät I - Bildungs- und Sozialwissenschaften, Institut für Sozialwissenschaften, Abteilung für politische Bildung und Politikdidaktik, Carl von Ossietzky Universität Oldenburg, Ammerländer Heerstraße 114-118, 26129 Oldenburg, Deutschland

E-Mail: antje.gansewig@uni-oldenburg.de

Dr. M. Walsh

Bonn, Deutschland

E-Mail: maria.walsh@gmx.de
} 
derheiten hin. So wurden neben den mehrheitlichen Positivbewertungen - die sich insbesondere auf die Person des Aussteigers und weniger auf die Maßnahmeninhalte bezogen - auch kritische Aspekte identifiziert. Einige Schüler/innen fühlten sich etwa aufgrund detaillierter Gewaltdarlegungen unwohl. Zudem wurden mitunter Geschlechterspezifika festgestellt. Resümierend verdeutlichen die Evaluationsresultate einen grundsätzlichen Modifizierungs- und Forschungsbedarf der Anwendungspraxis. Abschließend führen die Autorinnen Empfehlungen für Bildungsakteure und perspektivische Überlegungen an.

Schlüsselwörter Evaluationsstudie - Randomisiertes Kontrollgruppendesign · Schulische Extremismusprävention · Aussteiger/innen aus politisch extremen Szenen · Ehemalige Rechtsextreme · Biografiebasierte Schulmaßnahme

\title{
Students' view of a primary prevention seminar conducted by a former right-wing extremist in schools: Findings of an evaluation study and recommendations for educators
}

\begin{abstract}
The engagement of former right-wing extremists in school-based Prevention of Violent Extremism (PVE) has been taking place in Germany for two decades. Its main objective is the prevention of radicalization and extremism among students. However, it is remarkable that this particular format has to date rarely been empirically investigated. Thus, the (non-)intended effects of such interventions on students and their views remain largely unclear. In light of this, the authors conducted a comprehensive research project on the involvement of former extremists in schools, focusing on the students' perspective. It included a survey of the German status quo and the examination of an exemplary primary prevention seminar by way of an impact and process evaluation at 13 public community schools using a randomized controlled trial (50 classes). This paper presents and discusses certain relevant results of the evaluation, focusing on the student surveys regarding their assessment. The findings of the data collected directly after the school intervention $(n=490)$ and on average five and a half months later $(n=448)$ indicate a heterogeneous student perspective and specific factors. Thus, in addition to the mainly positive students' assessments-referring primarily to the former and less to the measure contents-we identified critical aspects. Some students e.g., felt uncomfortable because of detailed descriptions of violence. Furthermore, we noted gender differences. In summary, the results show that the current application practice is in need of a paradigm shift towards research-driven modifications. The article ends with recommendations for educators and an outlook on perspectives.
\end{abstract}

Keywords Evaluation study $\cdot$ Randomized controlled trial $\cdot$ Preventing violent extremism in schools · Formers · Former right-wing extremist · Biography-based school intervention 


\section{Stand der Forschung und Zielsetzungen des Beitrags}

Aufgrund der politischen und gesellschaftlichen Entwicklungen in den letzten Jahren hat die Auseinandersetzung mit Themen wie Demokratieerziehung sowie Prävention von Radikalisierung ${ }^{1}$ und politischem Extremismus ${ }^{2}$ in der Schule einen größeren Stellenwert erfahren. Gleichermaßen werden diese Themen komplexer und die von Lehrkräften zu vermittelnden Inhalte und an sie gestellten Anforderungen herausfordernder. Zur Vermittlung spezifischer (Fach-)Inhalte, emotionaler und sozialer Kompetenzen sowie demokratischer Werte, greifen Schulen ebenfalls auf externe Referent/innen zurück. In diesem Zusammenhang finden etwa biografiebasierte Maßnahmen von und mit Aussteiger/innen aus politisch extremen Szenen ${ }^{3}$ Anwendung. In Deutschland ist dies bereits seit den frühen 2000er Jahren der Fall. ${ }^{4}$ Hierbei standen und stehen ehemalige extrem Rechte im Vordergrund, seit einigen Jahren finden entsprechende Schulveranstaltungen genauso zu anderen Extremismusformen statt (vgl. Gansewig und Walsh 2018, 2020a). Oftmals sind Aussteiger/ innen mit diesen Tätigkeiten institutionell bei Ausstiegshilfen angebunden; einige von ihnen gehen diesen Aktivitäten jedoch auch in selbstständiger Form nach. Die zumeist primärpräventiv ${ }^{5}$ ausgerichteten Interventionen werden hauptsächlich von männlichen Aussteigern, in allen Schulformen und in der Regel ab der siebten Klassenstufe als einmalige Schulveranstaltungen durchgeführt. Dauer, Honorare, mediale Berichterstattung, Methodik und Inhalte - unabhängig von den individuellen Biogra-

\footnotetext{
${ }^{1}$ Nach Horgan (2009) ist Radikalisierung ,the social and psychological process of incrementally experienced commitment to extremist political or religious ideology" (S. 152).

2 Mit politischem Extremismus ist in diesem Beitrag eine fundamentale Demokratiefeindlichkeit gemeint, „die jeweils eine spezifische ideologische Ausprägung einnehmen“ und sowohl auf Einstellungs- als auch auf Handlungsebene sichtbar werden kann. Islamismus wird als eine ,religiös begründete Form des politischen Extremismus“" verstanden (Gansewig 2018, S. 465 ff.). Zudem werden politisch extreme Orientierungen und Erscheinungsformen als Phänomene betrachtet, die ebenso in der Mitte der Gesellschaft anzutreffen sind und ,sich nicht an äußeren politischen Rändern entwickeln und etablieren. Sie werden durch gesamtgesellschaftliche, ökonomische, soziale und politische Entwicklungen und Diskurse konstituiert und getragen." (Gansewig und Walsh 2020a, S. 41).
}

${ }^{3}$ Hierunter sind Personen zu verstehen, die über sich selbst sagen, dass sie aus einer politisch extremen Szene ausgestiegen seien. Nach Horgan (2009) erfolgt der Ausstieg prozesshaft und es bedarf der Unterscheidung zwischen Disengagement und Deradikalisierung. Während sich ersteres auf die Verhaltensebene (keine Zugehörigkeit zu einer politisch extremen Gruppe, Bewegung bzw. Szene) bezieht, wird unter Deradikalisierung die Einstellungsebene (keine Identifizierung mit einer politisch extremen Ideologie) verstanden. Disengagement muss dabei nicht notwendigerweise mit Deradikalisierung einhergehen (S. 140ff.). Wann von einem erfolgreichen Ausstieg gesprochen werden kann, wird kontrovers diskutiert. So erscheint neben der Verhaltensänderung ein entsprechender Wandel auch auf der Einstellungsebene erstrebenswert. Dieser ist jedoch herausfordernd in der Bearbeitung, Überprüfbarkeit und Legitimation vor dem Hintergrund der durch das Grundgesetz gesicherten Rechte der Meinungs- und Religionsfreiheit (Gansewig und Walsh 2020a, S. 55 f.).

${ }^{4}$ Auch in Österreich spielen entsprechende Maßnahmen eine Rolle (z.B. https://www.tagesschau. de/investigativ/panorama/is-rueckkehrer-113.html. Zugegriffen: 30.01.2021; https://kurier.at/chronik/ praevention-in-schulen-durch-gelaeuterte-is-anhaenger-steckt-fest/189.195.559. 30.01.2021).

Zugegriffen:

5 Primärprävention richtet sich an die Allgemeinheit und zielt auf die generellen Ursachen des zu prävenierenden Phänomens; Sekundärprävention knüpft an bereits erkennbare Risiken und Problemlagen an; Tertiärpräventive Maßnahmen hingegen wenden sich an bereits manifestierte Probleme (Meier 2010, S. 274f.). 
fien - der Offerten sind variabel. Die schulische Einbindung ehemaliger Extremist/ innen wird gemeinhin von Anbietenden, Fördernden und durchführenden Schulen damit begründet, dass biografische Erzählungen in ihrer aufklärerischen und abschreckenden Wirkung auf die Schüler/innenschaft besonders effektiv seien, da aus erster Hand erzählt wird. ${ }^{6}$ Durch ihren individuellen Erfahrungshintergrund sollen Aussteiger/innen den Schüler/innen insbesondere die Gefahren und Konsequenzen der jeweiligen Szenen verdeutlichen und somit letztlich primär- und gegebenenfalls sekundärpräventive Impulse setzen (vgl. Gansewig und Walsh 2020a, b).

Wie allerdings aus der Präventionswissenschaft hinlänglich bekannt, ist gut gemeint nicht zwangsläufig sinnvoll oder in der intendierten Weise wirksam (vgl. z. B. Petrosino et al. 2000). Inwiefern die (Wirk-)Annahmen und Zielstellungen der Schulmaßnahmen mit Aussteiger/innen den tatsächlichen Gegebenheiten entsprechen, ist bislang - auch international - bemerkenswerterweise nur marginal wissenschaftlich untersucht worden (vgl. Gansewig und Walsh 2020a, b; Scrivens et al. 2020). Eine differenzierte Betrachtung etwa der vermittelten Maßnahmeninhalte sowie eine gegenstandsangemessene Erhebung der Schüler/innenperspektiven erfolgte bislang nur punktuell. Infolgedessen konnten empirisch fundierte Aussagen zu einer kriminalpräventiven und/oder extremismusvorbeugenden Wirkung sowie zu möglichen nichtintendierten Effekten kaum getroffen werden. Zudem sind die Effekte auf (langfristige) Lernprozesse bei der Schüler/innenschaft weitestgehend unklar. Grundsätzlich sprechen zudem empirische Erkenntnisse etwa aus der schulischen Suchtprävention gegen die Durchführung biografiebasierter Maßnahmen. Diese gelten - insbesondere dann, wenn sie einen warnenden beziehungsweise abschreckenden Charakter haben sowie der Informationsvermittlung dienen - in Expertenkreisen als ,,im günstigsten Fall ineffektiv, im ungünstigsten Fall schädlich in Hinblick auf die Reduzierung eines Missbrauchsverhaltens“ (Künzel-Böhmer, Bühringer und Janik-Konecny 1993, S. 103; siehe auch EUSPR 2019; Walsh und Gansewig 2020).

Der vorliegende Beitrag setzt sich anhand der Sichtweise von Schüler/innen genauer mit dem Potenzial und den Herausforderungen biografiebasierter Maßnahmen früherer Extremist/innen auseinander und möchte somit einen Beitrag zu diesem Schwerpunktheft leisten, in dem Schüler/innen als Akteure von Schule im Mittelpunkt stehen. Zunächst wird ein Überblick zum Stand der Forschung gegeben, bevor auf wesentliche Ergebnisse einer Evaluationsstudie eingegangen wird. Weiterhin werden Empfehlungen für Bildungsakteure behandelt und abschließend ein Fazit gezogen, Limitationen benannt und perspektivische Überlegungen angestellt.

\footnotetext{
6 Z.B. https://www.bige.bayern.de/beratung_und_bildung/km_und_bige/vortraege_und_workshops_an_ schulen/index.html; https:/www.ln-online.de/Lokales/Luebeck/Luebecker-Kiwanier-foerdern-Unterrichtgegen-Extremismus; https://www.der-reporter.de/neustadt/neustadt-in-holstein/artikel/allein-kommtman-da-nicht-mehr-raus-maik-scheffler-aussteiger-aus-der-rechten-szene-besuchte-neustaedter-schulen. Zugegriffen jeweils: 30.01.2021.
} 


\section{Das Forschungsprojekt}

Vor dem Hintergrund der beschriebenen Wissens- und Forschungsdefizite setzten sich die Autorinnen erstmalig umfassend wissenschaftlich mit der schulischen Einbeziehung von Aussteiger/innen aus politisch extremen Szenen in Deutschland auseinander. Das großangelegte, empirische Forschungsprojekt ${ }^{7}$, dass die Schüler/innen von Beginn an in den Fokus stellte, umfasste zum einen eine Bestandsaufnahme zum schulischen Einsatz ehemaliger Extremist/innen, die sowohl auf semistrukturierten Interviews als auch auf schriftlichen Befragungen relevanter Akteur/innen sowie auf einer Medienanalyse basierte. Zum anderen wurde exemplarisch eine schulbasierte Präventionsmaßnahme eines ehemaligen Rechtsextremen evaluiert (vgl. Gansewig und Walsh 2020a, S. 124f.).

\subsection{Die Schulmaßnahme}

Bei der untersuchten primärpräventiven Intervention handelte es sich um eine vier Schulstunden (180 min) umfassende Lerneinheit, die von einem Aussteiger aus der extrem rechten Szene ab der achten Klassenstufe durchgeführt wird. Die Ziele bestehen darin, Rechtsextremismus, Gewalt und Kriminalität vorzubeugen. Ein Projektkonzept lag im Untersuchungszeitraum nicht vor. Nach eigenen Aussagen ging der ehemalige Rechtsextreme anhand eines selbst erstellten Ablaufplans vor. Während eingangs im Theorieteil die Themen historischer Nationalsozialismus in Deutschland, Rechtsextremismus und Kriminalität im Fokus standen, erfolgte im sich anschließenden Biografieteil unter anderem die Auseinandersetzung mit dem Themenkomplex Rechtsextremismus anhand der Punkte Hinwendung, Leben als Rechtsextremer und Distanzierung. Nachfolgend behandelte er seine Erfahrungen im Rotlicht- und kriminellen Rockermilieu, bevor er seine Haftzeit und sein derzeitiges Leben schilderte. Während der Intervention wendete der Referent verschiedene Methoden der Unterrichtsgestaltung wie etwa ein Rollenspiel und die Verwendung von Bild- und Videomaterial an. Zum Abschluss der Präventionsmaßnahme war gemäß Ablaufplan eine offene Diskussion vorgesehen (vgl. Gansewig und Walsh 2020a, S. $248 \mathrm{ff}$.).

Die Intervention orientierte sich inhaltlich vorrangig an der Biografie des im Jahr 1978 geborenen Aussteigers ${ }^{8}$. Laut eigener Aussage lebte er über 20 Jahre in diversen von Gewalt geprägten Subkulturen. Circa zehn Jahre lang war er dabei in unterschiedlichen rechtsextremen Bezügen aktiv, bevor er als Mitglied eines kriminellen Rockerclubs in der organisierten Kriminalität tätig wurde. In beiden Strukturen übernahm er führende Funktionen. Ursächlich für die Distanzierung von Rechtsextremismus und Kriminalität waren nach eigener Aussage vor allem Streitigkeiten

\footnotetext{
7 Das Forschungsprojekt wurde zwischen Frühjahr 2017 und Dezember 2019 am Nationalen Zentrum für Kriminalprävention durchgeführt.

8 Um weder die Person noch deren Präventionstätigkeiten zu bewerben oder zu diskreditieren, haben die Autorinnen von Beginn des Forschungsprojekts an davon abgesehen, den Aussteiger öffentlich namentlich zu benennen. Ihm selbst stand und steht es frei, sich namentlich mit den Ergebnissen der Evaluationsstudie in Verbindung zu bringen. Eine über die im Rahmen der Evaluation hinausgehende Zusammenarbeit zwischen dem Aussteiger und den Autorinnen bestand nicht.
} 
innerhalb der jeweiligen Subkultur sowie psychische und physische Beeinträchtigungen. Während der Verbüßung einer Haftstrafe von zwei Jahren und zehn Monaten begab er sich freiwillig in psychologische Behandlung, um seinen Ausstiegsprozess weiterzuführen. Insbesondere durch den Psychologen der Justizvollzugsanstalt wurde er ermuntert, sich nach der Haftentlassung etwa gegen Rechtsextremismus und Kriminalität einzusetzen. Seit der Haftentlassung im Januar 2016 unterbreitet er selbstständig beziehungsweise im Rahmen seines eigens dafür gegründeten Vereins, diverse Angebote für die Präventions- und Deradikalisierungsarbeit (ebd.). Insbesondere seit Frühjahr 2018 ist er hierbei auch in Funk und Fernsehen sowie in sozialen Medien äußerst präsent und berichtet detailliert hauptsächlich über seine Vergangenheit.

\subsection{Die Evaluationsstudie}

Da die ergebnisoffene Evaluationsstudie ${ }^{9}$ auch Rückschlüsse auf die Anwendungspraxis in diesem Bereich zulassen sollte, wurden durch die Evaluatorinnen keine Änderungen an der exemplarischen Schulmaßnahme vorgenommen. ${ }^{10}$ Ein wesentliches Ziel der Evaluationsstudie war es, Erkenntnisse zu Effekten von entsprechenden primärpräventiven Angeboten auf Schüler/innen zu generieren. Sechs Fragen standen im Zentrum des Interesses der Schüler/innenbefragungen:

- Wie ist die Sicht auf Präventionsmaßnahme, Referent und subjektiven Wissenszugewinn?

- Wird subjektiv von einer Einstellungs- und/oder Verhaltensänderung aufgrund der Intervention ausgegangen?

- Gibt es Anhaltspunkte für mögliche Faszinations- und (Re-)Traumatisierungsaspekte?

- Zeigen sich Veränderungen in den Variablen rechtsextreme Einstellungen, Gewalt und Delinquenz?

- Lassen sich ein Wissenszugewinn und/oder eine außerschulische Weiterbeschäftigung mit dem Thema Rechtsextremismus feststellen?

- Zeigen sich merkmalsbezogene Unterschiede (z.B. Geschlecht) bei den Schüler/ innenrückmeldungen?

Hierfür wurde eine methodenexterne Triangulationsstudie bestehend aus Voruntersuchung, Prozessevaluation und Wirkungsuntersuchung durchgeführt. Sie erfolgte im Schuljahr 2017/18 in 50 Schulklassen an 13 öffentlichen Gemeinschaftsschulen in Schleswig-Holstein. Die teilnehmenden Klassen wurden einer Experimental- (EG) und Kontrollgruppe (KG) als geclusterte Zufallsstichprobe zugeordnet (EG/KG: $n=25$ ) (vgl. Walsh und Gansewig 2019, S. $29 \mathrm{ff}$.).

\footnotetext{
9 Die Evaluationsstudie erfolgte zwischen Frühjahr 2017 und Mai 2019 als Kooperation zwischen dem Nationalen Zentrum für Kriminalprävention und dem Landespräventionsrat Schleswig-Holstein.

10 Im Sommer 2016 kontaktierte der ehemalige Rechtsextreme den Landespräventionsrat Schleswig-Holstein und berichtete von seinen (außer-)schulischen Aktivitäten, die er bereits zu diesem Zeitpunkt sowohl inner- als auch außerhalb von Schleswig-Holstein offerierte (Walsh und Gansewig 2019, S. 26).
} 
Den Zielsetzungen der Intervention gemäß sollten positive Veränderungen im Hinblick auf extrem rechte Einstellungen, Gewalt und Delinquenz bei den Schüler/ innen eintreten. Die Überprüfung dieser Ziele stand im Fokus der Wirkungsuntersuchung, auf deren empirisches und methodisches Vorgehen sowie Analyseverfahren an dieser Stelle aus Platzgründen nicht näher eingegangen werden kann (siehe hierzu ebd., S. 36 ff.). Die Prozessevaluation begleitete die Durchführung der Schulmaßnahme, nahm deren Rahmenbedingungen, die inhaltliche und methodische Gestaltung sowie referentenspezifische Aspekte in den Blick. Hierfür wurden die teilnehmenden Schüler/innen im unmittelbaren Anschluss an die Intervention und im Durchschnitt fünfeinhalb Monate nach der Maßnahme schriftlich befragt. ${ }^{11}$ Im Folgenden stehen eben diese Schüler/inneneinschätzungen und somit insbesondere diese Teilergebnisse der Prozessevaluation im Vordergrund. Sie generierten sich sowohl aus geschlossenen als auch aus offenen Frageformaten zu beiden Messzeitpunkten. Die Auswertung der geschlossenen Fragen erfolgte deskriptiv, die der offenen Fragen mittels Kategorisierungen (ausführlich hierzu Gansewig und Walsh 2020a, S. 270 f.).

\section{Ergebnisse der Evaluationsstudie aus Schüler/innenperspektive}

\subsection{Die Stichproben}

In die Auswertung der Prozessevaluation gingen unmittelbar nach der Intervention $\left(\right.$ MZP I) ${ }^{12}$ insgesamt 490 und im Durchschnitt fünfeinhalb Monate nach der Maßnahmenteilnahme (MZP II) 448 Schüler/innenfragebögen ein (Tab. 1). Der Anteil zwischen weiblichen und männlichen Befragten war jeweils ausgeglichen (MZP I: ㅇ: $48 \%, \sigma^{x}: 52 \%$; MZP II: ㅇ: $47 \%, \sigma^{7}: 53 \%$ ). Der Altersdurchschnitt beider Stichproben lag bei 15 Jahren (MZP I: $48 \%$; MZP II: 62\%); die jüngsten Teilnehmenden waren zum Zeitpunkt der Befragung 13 Jahre alt, die ältesten 17 Jahre. Die überwiegende Mehrheit (MZP I: 78\%; MZP II: 77\%) hatte keinen Migrationshintergrund (Gansewig und Walsh 2020a, S. 305 f.).

Ferner werden die folgenden Ausführungen teils durch Ergebnisse der Wirkungsuntersuchung ergänzt. Diese stützen sich auf die schriftliche Befragung von 564 Schüler/innen zum zweiten Messzeitpunkt (EG: $n=300$; KG: $n=264$ ) (Walsh und Gansewig 2019, S. 69 ff.).

\subsection{Generelle Schüler/inneneinschätzungen und Gesamtbewertung}

Sowohl die Präventionsmaßnahme als auch der Referent wurden von der Mehrheit der Schüler/innen unmittelbar nach der Intervention positiv beurteilt. So bewerteten

\footnotetext{
11 Bestandteile der Prozessevaluation waren zudem systematische, teilnehmende Beobachtungen in jeweils einer Klasse der sieben Schulen der Experimentalgruppe und schriftliche Befragungen der den Maßnahmen beiwohnenden Lehrkräfte sowie der Ansprechpartner/innen der Schulen (Gansewig und Walsh 2020a, S. 272 ff.).

12 Die Durchführung der Maßnahmen durch den Referenten in der EG fanden von November 2017 bis Februar 2018 statt (Gansewig und Walsh 2020a, S. 257).
} 
Tab. 1 Stichproben zu beiden Messzeitpunkten (Prozessevaluation)

\begin{tabular}{lllll}
\hline & MZP I & & MZP II \\
& $n$ & $\%$ & $n$ & $\%$ \\
\hline Geschlecht & & & & \\
Weiblich & 235 & 48 & 209 & 46,9 \\
Männlich & 253 & 51,6 & 236 & 52,4 \\
Keine Angabe & 2 & 0,4 & 3 & 0,7 \\
Alter & & & & \\
$<$ 15 Jahre & 179 & 36,5 & 57 & 13 \\
15 Jahre & 236 & 48,2 & 278 & 62 \\
$>$ 15 Jahre & 74 & 15,1 & 113 & 25 \\
Keine Angabe & 1 & 0,2 & - & - \\
Migrationshintergrund & & & & \\
Ja & 108 & 22 & 104 & 23 \\
Nein & 382 & 78 & 343 & 76,8 \\
Keine Angabe & - & - & 1 & 0,2 \\
Insgesamt & 490 & 100 & 448 & 100 \\
\hline & & & &
\end{tabular}

$90 \%$ der Schüler/innen den Referenten $(n=443)$ und $81 \%$ die Maßnahme $(n=402)$ auf einer Schulnotenskala mit sehr gut und gut, wobei in der Tendenz Schülerinnen wohlwollender benoteten. Als mangelhaft und ungenügend wurde sowohl die Intervention als auch der Referent ausschließlich von Jungen bewertet (Walsh und Gansewig 2019, S. 63 f.). Diese überwiegend positiven Einschätzungen zeigten sich auch in den Antworten auf die Fragen „Was hat Dir besonders gut/nicht gefallen an der Präventionsmaßnahme?“. 93\% aller Befragten $(n=457)$ beantworteten die Frage, was ihnen besonders gut gefallen habe. Hingegen äußerte sich lediglich die Hälfte dazu, was ihnen nicht gefallen habe $(n=243)$. 60 Schülerinnen und 46 Schüler nannten hierbei explizite Kritikpunkte. Wie die Betrachtung der Schüler/innenangaben zu positiven Faktoren der Präventionsmaßnahme aufzeigte, bezogen sich von den 464 detaillierten Nennungen 65,5\% auf Authentizitätsaspekte, den Habitus und die Vortragsweise des ehemaligen Rechtsextremen (z.B. „Das [sic] es einer war der das alles wirklich erlebt hat und somit man sich besser hineinversetzten [sic] konnte“; „Das [sic] [der Referent (Anm. d. Verf.)] ein chilliger und lockerer Typ ist“). Ferner bewerteten die Befragten die methodische Gestaltung mit $17 \%$ positiv. Eine deutlich untergeordnete Rolle spielten im Vergleich die Inhalte der Maßnahme und Lerneffekte, auf die lediglich $10 \%$ beziehungsweise $5 \%$ der Schüler/innenangaben abzielten. Hinsichtlich negativer Aspekte bemängelten $42 \%$ der Teilnehmenden bestimmte Rahmenbedingungen, wie etwa, dass die Präventionsmaßnahme zu kurz gewesen sei und es keine Pausen gegeben habe. Zudem missfielen einigen Schüler/ innen inhaltliche Komponenten (24\%), beispielsweise, dass nicht alle Inhalte intensiv behandelt worden seien und einzelne Themen ,teilweise vielleicht ein wenig zu extrem“ gewesen seien beziehungsweise es ,manchmal [...] bei dem Thema Gewalt zu sehr ins Detail“" ging. Den ehemaligen Rechtsextremen betreffende Beanstandungen $(19 \%)$ bezogen sich etwa auf das laute Sprechen und die Wortwahl (ebd., S. $51 \mathrm{ff}$.$) .$ 
Die vornehmlich positive Perspektive der Schüler/innen auf den Referenten spiegelte sich ferner in deren Angaben zu dessen Beschreibung mit einem Eigenschaftswort zum ersten Messzeitpunkt wider. Die angeführten 412 Adjektive wiesen zu $87 \%$ eine positive Konnotation auf (z.B. „offen“, „,cool“, „ehrlich“). Vier Schülerinnen und neun Schüler verwendeten hingegen Adjektive mit einer eindeutig negativen Konnotation, wobei sechs von ihnen den Aussteiger als ,laut" und eine Schülerin ihn etwa als ,angsteinflößend“ beschrieben (Gansewig und Walsh 2020a, S. $327 \mathrm{ff}$.).

Ferner wurden die Schüler/innen unmittelbar nach der Intervention gefragt, ob sie sich an einer Stelle der Präventionsmaßnahme unwohl gefühlt hatten. Dies bejahten $16 \%$ aller Befragten $(n=80)$ und mit $25 \%$ signifikant häufiger die Schülerinnen $\left(\sigma^{\top}\right.$ : 9\%; Pearsons $\left.\mathrm{Chi}^{2}(2)=21,721 ; p<0,001\right)$. Diese Unbehaglichkeitsgefühle wurden in 69 Fällen von 50 Schülerinnen und 18 Schülern spezifisch benannt. ${ }^{13}$ Mit $64 \%$ konzentrierten sich die Schüler/innenaussagen auf die Inhaltsebene und hierbei wiederum vorrangig auf die Inhalte mit Gewaltbezug. Insbesondere die Erzählungen zu einem von anderen Rechtsextremen begangenen Tötungsdelikt, die rund ein Viertel aller konkret genannten Gründe ausmachten und vorrangig von Schülerinnen angegeben wurden, führten zu Unbehagen. 16\% der Ursachen, die ebenso mehrheitlich von Mädchen angeführt wurden, bezogen sich auf die Vergangenheit, den Habitus und das äußere Erscheinungsbild des Aussteigers. Insgesamt vier Befragte verwendeten in ihren Angaben zum Inhalt beziehungsweise zum Referenten explizit das Wort „Angst“ (z.B. ,[...] so etwas zu hören bereitet einem Angst“; „Als er sich groß gemacht hatte und immer lauter wurde. Weil ich da ein bisschen Angst hatte“; „Manchmal machte der Mann mir Angst“) (Walsh und Gansewig 2019, S. 59 ff.). Im Lauf der Präventionsmaßnahme Unbehagen verspürt zu haben, mündete jedoch nicht in eine negative Gesamtbewertung des Aussteigers oder der Maßnahme. Auch diese Schüler/innen bewerteten den Referenten zu $95 \%$ und die Intervention zu $80 \%$ mit sehr gut und gut (ebd., S. 63 f.).

Aus den Angaben der Schüler/innen konnten insbesondere zwei Schlussfolgerungen gezogen werden: (1) Dass ein reines Abfragen von „Was hat Dir (nicht) gefallen?" und/oder die Vergabe von Noten als Feedback nicht ausreicht, um die Schüler/ innenperspektive auf ein derartiges Schulformat adäquat zu erfassen; (2) Dass mit der untersuchten Präventionsmaßnahme das Interesse der Schüler/innen weniger für den eigentlichen Präventionsgegenstand geweckt werden konnte, sondern sich dieses vornehmlich auf die Person des Szeneaussteigers bezog (Gansewig und Walsh 2020a, S. $359 \mathrm{ff}$.$) .$

Letztere Schlussfolgerung konnte durch weitere Evaluationsergebnisse untermauert werden: So zeigten etwa die Resultate der Wirkungsuntersuchung, dass sich die Maßnahmenteilnehmenden im Vergleich zur Kontrollgruppe seit der ersten Befragung nicht signifikant häufiger außerhalb der Schule über die Thematik Rechtsextremismus informiert hatten. Diese Aussage wurde in beiden Gruppen jeweils von etwa drei Viertel der Schüler/innen verneint (Gansewig und Walsh 2020a, S. 390f.). Des Weiteren gab die Hälfte der Schüler/innen $(n=231)$ zu Messzeitpunkt II an, dem Referenten in sozialen Netzwerken gefolgt zu sein; hierbei konnte ein signi-

13 Ein Schüler benannte zwei Gründe für sein Unbehagen. 
fikanter Geschlechterunterschied festgestellt werden (o: $63 \%$; $\sigma^{\pi}: 47,5 \%$; Pearsons $\left.\mathrm{Chi}^{2}(1)=10,426 ; p=0,001\right)$. Insgesamt $8 \%$ der Befragten nahmen hierüber Kontakt zu ihm auf (ebd., S. 343 f.). Dies spricht dafür, dass zwar das geweckte Interesse an der Person des ehemaligen Rechtsextremen bei einigen Schüler/innen über die Schulmaßnahme hinausging; hinsichtlich des Präventionsgegenstands hingegen gelang dies nicht.

\subsection{Veränderungs- und Lernprozesse}

Die im Rahmen der Wirkungsuntersuchung generierten Ergebnisse wiesen nicht auf einen Einfluss der untersuchten Schulmaßnahme auf rechtsextreme Einstellungen, Gewalt und Delinquenz hin (Walsh und Gansewig 2019, S. 69 ff.). Auch die überwiegende Mehrheit der Befragten (76\%; $n=338$ ) ging zum zweiten Messzeitpunkt nicht von Veränderungen ihrer Einstellungen und/oder ihres Verhaltens aufgrund der Intervention aus. Von denjenigen 82 Teilnehmenden, die entsprechende Veränderungen bejahten, lagen von 26 Schülerinnen und 33 Schülern Konkretisierungen vor: Zur Hälfte $(n=32)$ thematisierten sie Perspektivänderungen hinsichtlich Rechtsextremismus und hier überwiegend eine geänderte Sichtweise auf Anhänger der rechten Szene $(n=23)$. So äußerten sich beispielweise eine Schülerin und neun Schüler ihnen gegenüber aversiv (z. B. ,Das [sic] man jetzt [...] weiß man sollte solche Menschen meiden/nicht begegnen oder mit ihnen Kontakt haben"), wohingegen sechs Schülerinnen eine tolerantere beziehungsweise verständnisvollere Einstellung Szenemitgliedern gegenüber nannten (z.B. „Ich denke jetzt das [sic] sie mir eig. leid tun [sic] müssen, da sie da mitmachen und bei anderen fehler [sic] suchen die von ihren Fehlern ablenken, und dass sie mir Leid tun [sic], weil sie so viel Hass in sich haben."). Ferner bezogen sich die konkret geäußerten subjektiven Einstellungsund/oder Verhaltensänderungen zu 22\% auf spezifische Verhaltensänderungen wie etwa eine erhöhte Vorsicht und Wachsamkeit gegenüber rechten Personen(gruppen) (Gansewig und Walsh 2020a, S. 346ff.).

Zu Messzeitpunkt I gaben $80 \%$ der Schüler/innen an $(n=395)$, durch die Intervention etwas gelernt zu haben. Es zeigten sich signifikante Geschlechterunterschiede in der Einschätzung eines Lernzugewinns (Pearsons $\mathrm{Chi}^{2}(2)=12,551 ; p=0,002$ ), den $89 \%$ der weiblichen und $77 \%$ der männlichen Teilnehmenden annahmen. Im Durchschnitt fünfeinhalb Monate nach der Maßnahme lag der Anteil der Befragten, die einen subjektiven Lernzugewinn bejahten, bei 59\% $(n=265)$ (Walsh und Gansewig 2019, S. 64f.). Eine vertiefende lerntheoretische Untersuchung der Schüler/ innenantworten setzte sich inhaltsanalytisch mit der Möglichkeit des kognitiven und affektiven Lernens durch die Maßnahme auseinander (Stemmeler 2020). Im Ergebnis zeigte sich hier eine Hemmung kognitiver Lernprozesse durch (1) mangelndes Vorwissen auf Seiten der Schüler/innen und (2) die zu undifferenzierte Darstellung der Diversität subkultureller Verhaftungen in der Biografie des Referenten. Mit Blick auf affektives Lernen wies die Untersuchung zwar auf Potenzial der Maßnahme hin, welches jedoch etwa durch das Fehlen einer kontinuierlichen, schulischen Beschäftigung mit dem Thema ungenutzt blieb (siehe hierzu auch DVPB 2018). Darüber hinaus war in Teilen eine Übertragung auf die Lebenswelt der Schüler/innen aufgrund des hohen Abstraktionsniveaus offenbar erschwert. Zwar schienen affektive 
Lerninhalte insgesamt in die angestrebte Richtung zu gehen; jedoch gab es - wie bei den zuvor angeführten subjektiven Einstellungsänderungen - auch hier Anzeichen für Empathieentwicklungen für Szeneanhänger (z.B. ,,[D]as [sic] sie nicht immer unbedingt böse sind“; Stemmeler 2020), was für ein Risiko von Verharmlosungstendenzen sprechen könnte (Gansewig und Walsh 2020a, S. 363). Ebenso ergaben die Ergebnisse der Wirkungsuntersuchung keine signifikanten Unterschiede hinsichtlich eines Wissenszugewinns der Interventionsteilnehmenden im Vergleich zur Kontrollgruppe. Die Analyse der offenen Fragen zu Ein- und Ausstiegsmotiven zeigte allerdings Gruppenunterschiede auf: So nannten die Maßnahmenteilnehmenden verschiedene mögliche Beweggründe für einen Szeneein- sowie -ausstieg, die in Verbindung mit der Präventionsmaßnahme gebracht werden konnten und lehnten ihre Antworten teils an die Biografie des Referenten an. Die Schüler/innen der Kontrollgruppe hingegen benannten vornehmlich ideologische Ursachen (ebd., S. $390 \mathrm{ff}$.$) .$

\subsection{Mögliche Faszinations- und (Re-)Traumatisierungsaspekte}

Mögliche Faszinationsaspekte hinsichtlich einer rechten und/oder kriminellen Lebensweise konnten in den Schülerdatenquellen nicht eruiert werden. ${ }^{14}$ So waren beispielsweise weder bei den Aussagen zu einer kriminellen Lebensweise noch bei denjenigen zum Leben in der rechten Szene signifikante Unterschiede zwischen der Experimental- und Kontrollgruppe festzustellen (Gansewig und Walsh 2020a, S. $400 \mathrm{f}$.). Ebenso lagen keine Anhaltspunkte für eventuelle Traumatisierungsaspekte bei Teilnehmenden mit Migrationshintergrund als potenziell Betroffene rechter Gewalt und Aspekte von Retraumatisierung von bereits Betroffenen vor (ebd., S. 363 f.).

Auf Grundlage der Resultate, die nicht auf eine (Re-)Traumatisierungsproblematik bei (potenziell) Betroffenen rechter verbaler und/oder physischer Gewalt hinweisen, sollte jedoch keinesfalls abgeleitet werden, dass (Re-)Traumatisierung in diesem speziellen Präventionskontext ein grundsätzlich zu vernachlässigendes Thema darstellt. So wurde die zugrundeliegende Evaluation - im Gegensatz zur gängigen Praxis beim Einsatz von biografiebasierten Schulinterventionen - nicht als Pflichtveranstaltung durchgeführt und eine Teilnahme erfolgte ausschließlich mit Einverständnis der Erziehungsberechtigten. Darüber hinaus gab es - unabhängig von einer Betroffenheit von rechter Gewalt - Anhaltspunkte für mögliche Retraumatisierungsaspekte bei einzelnen Schüler/innen: So begründete sich bei sieben Schülerinnen und einem Schüler die Unbehaglichkeit während der Maßnahme in ihrem eigenen Erfahrungshintergrund (z.B. „An der Stelle wo er über Mobbing gesprochen hat und in mir wieder alles hoch kam [sic] was so passiert ist") (ebd., S. $319 \mathrm{ff} ., 364)$.

\footnotetext{
14 Im Gegensatz hierzu benannten drei der den Maßnahmen beiwohnenden 28 Lehrkräfte eventuelle Begeisterungs- und Faszinationsaspekte: „So könne einiges bei manchen Schülern ,durchaus auch Begehrlichkeiten` geweckt beziehungsweise ,teilweise eher Anregungen zum Nachahmen` geboten haben. Zwei Lehrkräfte spezifizierten hierbei die Themenbereiche: ,Das Vorhandensein von Geld, Ansehen und Macht war für einige Schüler attraktiv. Daher fehlte mir der Tiefgang in den ,komplizierten' Stationen seines Lebens. ‘; ,Einige SuS himmeln Tattoos u. Gewaltbereiche an“." (Gansewig und Walsh 2020a, S. 301).
} 


\section{Empfehlungen für Bildungsakteure}

In Anbetracht des begrenzten Forschungsstands zu biografiebasierten Maßnahmen sowie der Ergebnisse des Forschungsprojekts bei gleichzeitig reger und jahrzehntelanger Einbindung von Szeneaussteigern in die schulische Präventionsarbeit, bestand eines der Projektziele darin, Empfehlungen für die Praxis zu generieren. Hierbei handelte es sich um ein Novum im deutschsprachigen Raum. Diese werden im Folgenden zusammenfassend skizziert; sie verstehen sich als Hinweise für eine zielgruppenorientierte Umsetzung, stellen jedoch keinen Garant für das Erreichen kriminal- und/oder extremismuspräventiver Effekte dar (vgl. Gansewig und Walsh 2019a, b).

Grundsätzlich ist es ratsam, sich im Vorfeld eine solide Informationsbasis (z.B. Seriosität des Angebots, Konzept, Qualifikation der/des Durchführenden) zu schaffen und Finanzierungsaspekte sowie mediale Darstellungen zu reflektieren (vgl. Gansewig und Walsh 2019a, S. 5 ff.). Sollte eine Entscheidung zugunsten der Durchführung einer Maßnahme einer/eines früheren Extremistin/Extremisten an der Schule fallen, ist es bedeutsam, diverse Punkte zu beachten. Diese fokussieren insbesondere die Rahmenbedingungen der schulischen Intervention, deren Inhalte und Methodik sowie Aspekte, die die/den Referierende/n betreffen (Abb. 1).

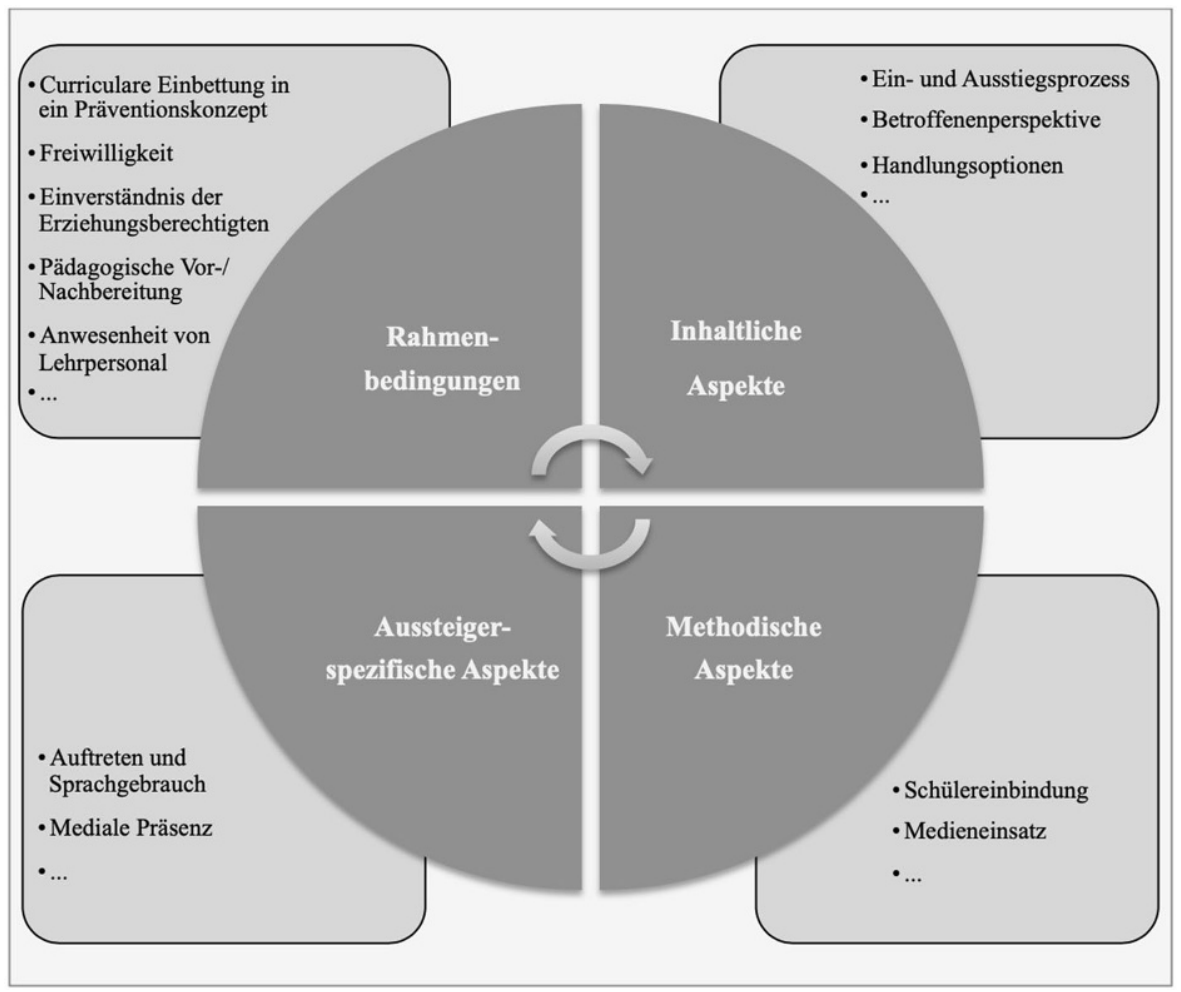

Abb. 1 Empfehlungen zur Umsetzung einer schulischen Intervention von und mit ehemaligen Extremist/ innen 
Im Sinne der Zweckhaftigkeit für die Schüler/innen sowie deren Wohlergehen sollte die Teilnahme - etwa aufgrund möglicher (Re-)Traumatisierungsaspekte zwingend auf Freiwilligkeit beruhen und ausschließlich mit dem Einverständnis der Erziehungsberechtigten möglich sein. Ebenso gilt es, klassen(stufen)übergreifende (Groß-)Veranstaltungen $\mathrm{zu}$ vermeiden, da diese keine gegenstandsangemessenen Präventionsmaßnahmen darstellen. Zielführend erscheint dagegen insbesondere für die Beteiligung der Kinder und Jugendlichen eine Umsetzung im Klassenverband. Aufgrund der Notwendigkeit der Kontextualisierung einer solchen schulischen Intervention ist von einer Durchführung vor der neunten Jahrgangsstufe abzusehen, wobei grundsätzlich stets eine Einzelfallentscheidung auf Basis der jeweiligen Klassenkonstitution, etwa in Bezug auf unterschiedliche Lernniveaus und Inklusionsaspekte, anzuraten ist. Als erforderlich werden zudem die verbindliche pädagogische Vor- und Nachbereitung erachtet, in denen sowohl inhaltliche als auch referentenbezogene Aspekte zu berücksichtigen sind. Auf die durchgängige Anwesenheit von Lehrpersonal im Lauf der Maßnahme sowie eine angemessene Pausengestaltung sollte ebenfalls nicht verzichtet werden (vgl. Gansewig und Walsh 2019a, S. $8 \mathrm{ff}$.). Der inhaltliche Fokus der Intervention sollte auf der Biografie der/des Referierenden liegen und die Prozesshaftigkeit von Ein- und Ausstieg verdeutlichen. Empfohlen wird zudem, die Betroffenenperspektive aufzugreifen und Handlungsalternativen für eine Hinwendung zu und Handlungsoptionen für eine Abwendung von politisch extremen Bezügen aufzuzeigen. Zu vermeiden sind detaillierte Erzählungen zu kriminellen und gewalttätigen Handlungen (ebd., S. 12). Als wesentlich werden außerdem eine angemessene Schüler/inneneinbindung sowie eine ansprechende methodische Ausgestaltung der Intervention angesehen, ohne dabei Propagandamaterial zu präsentieren (ebd., S. 13). Ratsam ist in diesem Zusammenhang auch, sich im Vorfeld einen persönlichen Eindruck von der jeweils durchführenden Person etwa hinsichtlich des Auftretens und des Sprachgebrauchs zu machen sowie inhaltliche und gestalterische Aspekte der Maßnahme zu erörtern (vgl. Gansewig und Walsh 2019a, S. 13 ff.). Generell gilt es zu beachten, dass ,,[d]ie beeindruckende Wirkung“ von Aussteiger/innen auf die Schüler/innenschaft immer auch negative Effekte, wie etwa eine Faszination, nach sich ziehen könnte (ebd., S. 15).

\section{Fazit und Diskussion}

Die Schüler/innenrückmeldungen zur untersuchten primärpräventiven Maßnahme eines früheren Rechtsextremen weisen sowohl auf positive als auch auf kritische Aspekte hin. ${ }^{15}$ Offenbar gelang es dem Referenten zur Mehrheit der Schüler/ innenschaft einen Zugang herzustellen. Insofern könnte die Intervention die Möglichkeit bieten, die Teilnehmenden in einer altersbedingt weniger zugänglichen Lebensphase zu erreichen. Ferner könnte der Maßnahme Potenzial für Modelllernen zukommen. Prinzipiell geht dies mit dem Risiko einher, bei fragwürdigen Maßnahmeninhalten und inadäquater Umsetzung unerwünschtes beziehungsweise

15 Auch die Erkenntnisse der teilnehmenden Beobachtungen bestätigen diese Resultate (Gansewig und Walsh 2020a, S. 272 ff.). 
kontrainduziertes Lernen zu bedingen. Vor diesem Hintergrund sollten die vorrangig positiven Beurteilungen der Schüler/innen nicht dazu veranlassen, kritische Aspekte zu vernachlässigen: Zum einen bezogen sich diese Positiveinschätzungen nicht auf die Inhaltsebene, sondern hauptsächlich auf die Person des Aussteigers; zum anderen zeigen die Studienergebnisse weder intendierte Lernzugewinne auf, noch sprechen sie für die Erreichung des Präventionsziels und bieten insofern keinen Anlass für Euphemismen. ${ }^{16}$ Eine differenzierte und objektive Betrachtung beziehungsweise Darstellung von Evaluationsergebnissen erscheint generell und gerade bei diesem sensiblen Format unabdingbar. Bleibt dieser verantwortungsvolle Umgang - gerade von Anbietenden - aus, hinterlässt das mehr als nur einen zweifelhaften Beigeschmack. Insbesondere Inhalte, die bei den Teilnehmenden zu Unbehagen geführt haben, müssten hinterfragt werden.

Mit Blick auf die Erreichung von Lerneffekten wurden weitere kritische Gesichtspunkte identifiziert: Wie der Gruppenvergleich der Beantwortung möglicher Ein- und Ausstiegsmotive hinsichtlich der rechten Szene erbrachte, benannten die Schüler/innen der Experimentalgruppe hier mitunter Beweggründe, die mit der Biografie des Aussteigers in Zusammenhang standen. Die Schüler/innenschaft der Kontrollgruppe konzentrierte sich hingegen eher auf ideologische Ursachen. Wie die teilnehmenden Beobachtungen aufzeigten, thematisierte der Referent kaum politische Ideologieaspekte in der Auseinandersetzung mit seinem Ein- und Ausstieg. Seine Distanzierung von der extrem rechten Szene kommunizierte er als fließenden Übergang in die organisierte Kriminalität, weshalb seine Darstellung des eigentlichen Ausstiegs, also der Abwendung von der kriminellen Rockergruppierung, die sich auch aus Mitgliedern seiner rechtsextremen Kameradschaft zusammensetzte, vornehmlich im Zusammenhang mit Kriminalität stand. Das unterschiedliche Antwortverhalten der beiden Gruppen könnte darauf hinweisen, dass die Antworten der Schüler/innen der Experimentalgruppe durch die Maßnahmeninhalte beeinflusst wurden und es ihnen insofern schwerfiel, die angeführte Biografie als lediglich ein Beispiel wahrzunehmen. Dementsprechend könnte dieses Ergebnis für Verallgemeinerungstendenzen sprechen (vgl. Gansewig und Walsh 2020a, S. 413f.). Wie die Ergebnisse von Bertrams Studie zum Einsatz von Zeitzeugen in der Bildungsarbeit (vgl. Bertram 2017, S. 111 ff.) könnten auch diese Ergebnisse darauf hindeuten, dass reale Begegnungen im Schulunterricht die kritische Reflexion des Gehörten hemmen können. Um eine solche Hemmung zu vermeiden, größere emotionale Distanz zu gewährleisten, transportierte Inhalte überprüfbar zu machen und damit kritische Aspekte auszuschließen, könnte video- oder textbasiertes biografisches Arbeiten eine dienliche Alternative darstellen (Gansewig und Walsh 2020a, S. 422; Bertram 2017). Darüber hinaus gelang es offenbar nicht, die Schüler/innen zu einer weiteren

\footnotetext{
16 Bedauerlicherweise findet teilweise eine selektive Darstellung der Studienresultate statt (z. B. https:// www.lpr.nrw.de/aufgaben/alle-meldungen/aktuelle_meldung_202002_Fachtagung/Vortrag-Armborst/ Folien_NZK_KKP_NRW.pdf, S. 31. Zugegriffen: 30.01.2021). Auch der Referent bewirbt etwa auf dem Internetauftritt seines Vereins in einem Beitrag vom 05.08.2020 seine Maßnahme anhand einer pauschalisierten und einseitigen Positivdarstellung der Studienergebnisse, ohne diese zur Einsicht zur Verfügung zu stellen (,Der ,Präventionsunterricht Rechtsextremismus' ist in einer großen Studie des Landes Schleswig-Holstein und des Nationalen Zentrums für Kriminalitätsprävention [sic] evaluiert und von Schülern als gut und sinnvoll bewertet worden.“ Zugegriffen: 30.01.2021).
} 
Auseinandersetzung mit dem eigentlichen Präventionsthema außerhalb der Schule $\mathrm{zu}$ veranlassen. Ein nachhaltiges Interesse blieb aus (siehe hierzu auch Lodenius 2010).

Weiterhin zeigten die Resultate der Evaluationsstudie, dass die Intervention beziehungsweise der Aussteiger bei 16\% der Schüler/innen negative Emotionen auslösten. Auch die im Forschungsprojekt durchgeführte Medienanalyse verweist bei Schulmaßnahmen mit ehemaligen Extremist/innen auf ein entsprechendes Potenzial (vgl. Gansewig und Walsh 2020a, S. 208 ff.). Insofern gilt es Rahmenbedingungen zu schaffen, die den Schüler/innen adäquate Handlungsmöglichkeiten bieten: z. B. freiwillige Teilnahme, Einbezug der Erziehungsberechtigten, Durchführung von Pausen, Vermeidung detaillierter Gewaltdarstellungen und unsensibler Sprache. Die Ergebnisse verdeutlichen ferner die Notwendigkeit einer generellen Beachtung von möglichen unerwünschten beziehungsweise kontrainduzierten Effekten: (1) (Re-)Traumatisierungsaspekte können nicht nur im Hinblick auf (potenziell) Betroffene rechter Gewalt und Faszinationsaspekte nicht ausschließlich hinsichtlich der rechten Szene und/oder einer kriminellen Lebensweise, sondern unabhängig vom eigentlichen Präventionsthema eine Rolle spielen. Sie könnten selbstverständlich auch immer dann auftreten, wenn etwa diskriminierende Aussagen oder ausführliche Gewaltdarstellungen gegenständlich sind. Hinsichtlich Retraumatisierungsaspekten gilt dies nicht nur für direkt, sondern auch für indirekt von körperlicher und/oder psychischer Gewalt betroffene Schüler/innen; (2) Begeisterungstendenzen für die Person des Durchführenden können nicht ausgeschlossen werden. Dies ist unter anderem problematisch, wenn sich Referent/innen nicht ihrer Vorbildrolle entsprechend verhalten, sowohl während der Intervention als auch im Rahmen ihrer Präsenzen in sozialen Medien - gerade wenn diese am Ende der Schulmaßnahme als Möglichkeit der Kontaktaufnahme angepriesen werden. Fragwürdige Inhalte und Verhaltensweisen sowie ein zweifelhafter Sprachgebrauch sind folglich nicht nur im Klassenzimmer zu vermeiden (vgl. Gansewig und Walsh 2020b). Personen, die beispielweise regelmäßig ihren Alkohol- und (E-)Zigarettenkonsum medial zur Schau stellen, müssen sich mit der Frage nach ihrer generellen Eignung für die Arbeit mit Kindern und Jugendlichen konfrontieren lassen.

Resümierend wird angemerkt, dass internationale Forschungserkenntnisse zur schulischen Extremismus-, Gewalt- und Kriminalprävention, auf die Überlegenheit von Maßnahmen zur Förderung sozialer Kompetenzen, die Einführung verbindlicher Verhaltensregeln und die Schulentwicklungsarbeit gegenüber rein wissensvermittelnden Informationsveranstaltungen mit aufklärendem und sensibilisierendem Charakter hinweisen. Nachhaltige Präventionsarbeit bedarf dementsprechend zum einen einer evidenzorientierten Ausrichtung ${ }^{17}$ (z. B. Gansewig und Walsh 2020a, b; Walsh und Gansewig 2020), zum anderen einer curricularen und schulischen Einbettung, im Idealfall in ein geprüftes Präventionskonzept. Präventionsthemen etwa einmal im Schuljahr aufzugreifen, dürfte insofern nicht in eine tatsächliche Vorbeugung unerwünschter Einstellungen und Verhaltensweisen münden (vgl. Gansewig und Walsh 2019a, S. 5). Des Weiteren vermag schulische Prävention von Radika-

17 Also einer Ausrichtung an fundierten empirischen Ergebnissen beziehungsweise am „beste[n] verfügbare[n] Wissen“ (Bromme et al. 2014, S. 9; vgl. Walsh 2020, S. 26). 
lisierung und politischem Extremismus selbstverständlich eine in Regelstrukturen fest verankerte politische Bildung der Schüler/innen nicht zu ersetzen (siehe hierzu DVPB 2018). Für biografiebasierte Präventionsmaßnahmen gilt zudem, dass hier lediglich eine exemplarische und subjektiv dargestellte Biografie und keine verallgemeinerbaren (Sach-)Informationen vermittelt werden. Die alleinige Behandlung einer Einzelbiografie wird somit weder der Komplexität der Themen Radikalisierung und (Rechts-)Extremismus noch dem Anspruch an eine effektive Präventionsarbeit gerecht. Insgesamt bedarf es demgemäß eines Bewusstseins dafür, dass biografiebasiertes Arbeiten seitens der Lehrkräfte, der Schüler/innen sowie der durchführenden Person/en zahlreiche Kompetenzen erfordert und generellen Herausforderungen unterliegt (vgl. Gansewig und Walsh 2020a).

\section{Limitationen und Ausblick}

Wie jedes Forschungsprojekt unterlag auch dieses Einschränkungen (vgl. Walsh und Gansewig 2019, S. 30 f.). Mit Blick auf die hier aufgeführten Evaluationsergebnisse sei insbesondere angeführt, dass sie an die Person des Aussteigers sowie die untersuchte Schulmaßnahme gebunden sind. Weiterhin ist nicht auszuschließen, dass die freiwillige Teilnahme sowie das Einholen des Einverständnisses der Erziehungsberechtigten Auswirkungen auf die Teilnahmebereitschaft einzelner rechtsaffiner Jugendlicher beziehungsweise solcher aus rechtsaffinen Elternhäusern mit sich brachte (ebd.). Gleichwohl konnte das Forschungsprojekt, das sich erstmals umfassend wissenschaftlich mit ehemaligen Extremist/innen in der schulischen Präventionsarbeit in Deutschland auseinandersetzte, zentrale Argumentationen empirisch hervorbringen, die die bis dato begrenzte Diskussion nachhaltig bereichern dürften. Ebenso konnte beispielsweise empirisch aufgezeigt werden, dass (1) einzelne kritische Aspekte, die in der untersuchten exemplarischen Schulmaßnahme zu Tage traten, auch bei Veranstaltungen, die von anderen ehemaligen Extremist/innen durchgeführt werden, eine Rolle spielen und (2) teils deutliche genderspezifische Rezeptionen existieren. Insgesamt zeigen die Resultate wesentliche Modifizierungs- und Forschungsbedarfe der Anwendungspraxis und hierbei insbesondere das Erfordernis einer Fokusverschiebung auf die Hauptzielgruppe - die teilnehmenden Kinder und Jugendlichen und einer Berücksichtigung von deren Heterogenität auf (vgl. Gansewig und Walsh 2020a, b). Bei der Durchführung schulischer Prävention sollte in erster Linie das Wohlergehen der Schüler/innenschaft im Vordergrund stehen. Positive Effekte von Präventionsformaten allgemein und für alle Schüler/innen gleichermaßen vorauszusetzen, ohne diese Annahmen etwa mittels belastbarer Wirkungsuntersuchungen zu prüfen, oder auch Feedback von Schüler/innen gegenstandsunangemessen zu erheben beziehungsweise heranzuziehen, ohne diese Rückmeldungen einer differenzierten und tiefergehenden Betrachtung zu unterziehen, erscheint insofern fragwürdig und steht im Gegensatz zum eigentlichen Präventionsgedanken (Gansewig und Walsh 2020a, S. $423 \mathrm{ff}$.).

Die empirische Überprüfung von Lernprozessen und deren Ergebnissen ist ein kontrovers diskutiertes Thema, das im letzten Jahr beispielsweise auch in einem Sonderheft des Journal of Social Science Education adressiert wurde (Szukala und 
Davies 2020). Szukala und Davies schlagen darin eine pragmatische Sichtweise auf diesen Diskurs vor (2020, S. 3). Ohne die Offenheit und Heterogenität von Bildungsprozessen in Frage stellen zu wollen, plädieren auch wir für eine Perspektive von „hohe[r] Realitätsorientierung und ausgeprägte[m] Pragmatismus“, wie sie bereits von Bromme und anderen (2014, S. 15) beschrieben wurde. Auch wir vertreten die Auffassung, dass insbesondere die Offenheit und Komplexität von Bildungsprozessen die Überprüfung von Bildungsangeboten unabdingbar machen (vgl. Bromme et al. 2014). Wie bereits erwähnt, können nichtintendierte Effekte keinesfalls grundsätzlich und/oder für jede Schülerin und jeden Schüler negiert werden. Unabhängig von den akademischen Diskursen zu Erziehung, Bildung und Prävention in der geisteswissenschaftlich orientierten Pädagogik finden in der Praxis Maßnahmen (auch von nicht pädagogisch geschultem Personal) statt und beeinflussen - bei aller Offenheit von Bildungsprozessen - die Entwicklung von Schülerinnen und Schülern. Eine wissenschaftliche Auseinandersetzung mit diesen Interventionen im Sinne einer problemorientierten empirischen Bildungsforschung (ebd., S. 9) ist aus unserer Sicht dementsprechend unabdingbar. Weiterhin möchten wir anregen, die Überprüfbarkeit von Teilergebnissen im Bereich des Wissens- und/oder Kompetenzzugewinns nicht als grundsätzlichen Widerspruch zur Offenheit von individuellen Bildungsprozessen zu sehen, sondern sie vielmehr - im Sinne Prenzels (2012) - als eine Aufgabe der problemorientierten, empirischen Bildungsforschung zu verstehen.

Abschließend soll nicht unerwähnt bleiben, dass die Generierung empirischer Evidenz nur dann zweckmäßig ist, wenn sie sich in der Anwendungspraxis angemessen niederschlägt. Bedauerlicherweise ist dies jedoch nicht immer der Fall (vgl. Walsh und Gansewig 2020). Die Gründe hierfür sind vielfältiger Natur. Eine der Ursachen liegt sicherlich darin begründet, dass - wie Döring (2014) zutreffend ausführt - die Veröffentlichung von Evaluationsergebnissen und Praxisempfehlungen in Berichten für eine Optimierung und evidenzgeleitete Entscheidungsfindung in der Regel nicht ausreichend ist: „Denn oft kann nicht davon ausgegangen werden, dass Evaluationsberichte auch wirklich gelesen, verstanden und akzeptiert werden und dann auch noch zur Ableitung und Umsetzung der richtigen Konsequenzen führen.“ (Döring 2014, S. 173). Hier gilt es zukünftig, das Zusammenwirken von Wissenschaft, Praxis und Politik zu verbessern, um die Präventionsbemühungen an Schulen im Sinne der Schüler/innen gemeinsam zu optimieren.

Funding Open Access funding enabled and organized by Projekt DEAL.

Open Access Dieser Artikel wird unter der Creative Commons Namensnennung 4.0 International Lizenz veröffentlicht, welche die Nutzung, Vervielfältigung, Bearbeitung, Verbreitung und Wiedergabe in jeglichem Medium und Format erlaubt, sofern Sie den/die ursprünglichen Autor(en) und die Quelle ordnungsgemäß nennen, einen Link zur Creative Commons Lizenz beifügen und angeben, ob Änderungen vorgenommen wurden.

Die in diesem Artikel enthaltenen Bilder und sonstiges Drittmaterial unterliegen ebenfalls der genannten Creative Commons Lizenz, sofern sich aus der Abbildungslegende nichts anderes ergibt. Sofern das betreffende Material nicht unter der genannten Creative Commons Lizenz steht und die betreffende Handlung nicht nach gesetzlichen Vorschriften erlaubt ist, ist für die oben aufgeführten Weiterverwendungen des Materials die Einwilligung des jeweiligen Rechteinhabers einzuholen. 
Weitere Details zur Lizenz entnehmen Sie bitte der Lizenzinformation auf http://creativecommons.org/ licenses/by/4.0/deed.de.

\section{Literatur}

Bertram, C. (2017). Zeitzeugen im Geschichtsunterricht. Chance oder Risiko für historisches Lernen? Eine randomisierte Interventionsstudie. Schwalbach/Ts.: Wochenschau.

Bromme, R., Prenzel, M., \& Jäger, M. (2014). Empirische Bildungsforschung und evidenzbasierte Bildungspolitik. Eine Analyse von Anforderungen an die Darstellung, Interpretation und Rezeption empirischer Befunde. Zeitschrift für Erziehungswissenschaften, 17, 3-54.

Bundesvorstand der Deutschen Vereinigung für Politische Bildung (DVPB) (2018). Appell der Deutschen Vereinigung für Politische Bildung. Demokratie-Auftrag von Schule erfüllen, Politische Bildung stärken! Hannover: DVPB.

Döring, N. (2014). Evaluationsforschung. In N. Baur \& J. Blasius (Hrsg.), Handbuch Methoden der empirischen Sozialforschung (S. 167-181). Wiesbaden: Springer VS.

European Society for Prevention Research (EUSPR) (2019). Position der Europäischen Gesellschaft für Präventionsforschung zu ineffektiven und potenziell schädlichen Ansätzen in der Suchtprävention. Palma: EUSPR.

Gansewig, A. (2018). Prävention von politischem Extremismus in Deutschland. Eine Betrachtung zur Bedarfs- und Angebotslage. In M. Walsh, B. Pniewski, M. Kober \& A. Armborst (Hrsg.), Evidenzorientierte Kriminalprävention in Deutschland. Ein Leitfaden für Politik und Praxis (S. 465-488). Wiesbaden: Springer.

Gansewig, A., \& Walsh, M. (2018). Ehemalige Extremisten in der Präventionsarbeit. Eine bundesweite Erhebung zu Bestand und antizipierter Wirksamkeit. forum kriminalprävention, 4, 17-22.

Gansewig, A., \& Walsh, M. (2019a). Ehemalige Rechtsextreme in der schulischen Präventions- und Bildungsarbeit. Empfehlungen für Bildungsakteure. Bonn: Nationales Zentrum für Kriminalprävention.

Gansewig, A., \& Walsh, M. (2019b). Schulbasierte Präventionsmaßnahmen von Aussteigern aus der rechtsextremen Szene. Informationsbroschüre für Akteure der Bildungsarbeit. Kiel: Landespräventionsrat Schleswig-Holstein.

Gansewig, A., \& Walsh, M. (2020a). Biografiebasierte Maßnahmen in der schulischen Präventions- und Bildungsarbeit. Eine empirische Betrachtung des Einsatzes von Aussteigern aus extremistischen Szenen unter besonderer Berücksichtigung ehemaliger Rechtsextremer. Baden-Baden: Nomos.

Gansewig, A., \& Walsh, M. (2020b). Aussteiger aus extremistischen Szenen in der Schule. Ein Plädoyer für eine Fokusverschiebung auf die Schülerperspektive und deren differenzierte Betrachtung. In M. Walsh \& A. Gansewig (Hrsg.), Frühere Extremisten in der schulischen Präventionsarbeit. Perspektiven aus Wissenschaft und Praxis. Berichte des Nationalen Zentrums für Kriminalprävention, Nr. 1/2020. (S. 23-35). Bonn: Nationales Zentrum für Kriminalprävention.

Horgan, J. (2009). Walking away from terrorism: Accounts of disengagement from radical and extremist movements. New York: Routledge.

Künzel-Böhmer, J., Bühringer, G., \& Janik-Konecny, T. (1993). Expertise zur Primärprävention des Substanzmißbrauchs. Baden-Baden: Nomos.

Lodenius, A.-L. (2010). To leave a destructive life full of hate. The Story of Exit in Sweden.

Meier, B.-D. (2010). Kriminologie (4. Aufl.). München: C. H. Beck.

Petrosino, A., Turpin-Petrosino, C., \& Finckenauer, J. (2000). Well-meaning programs can have harmful effects! Lessons from experiments from programs such as scared straight. Crime \& Delinquency, 46(3), 354-379.

Prenzel, M. (2012). Empirische Bildungsforschung morgen: Reichen unsere bisherigen Forschungsansätze aus? In M. Gläser-Zikuda, T. Seidel, C. Rohlfs, A. Gröschner \& S. Ziegelbauer (Hrsg.), Mixed Methods in der empirischen Bildungsforschung (S. 273-286). Münster: Waxmann.

Scrivens, R., Windisch, S., \& Simi, P. (2020). Former extremists in radicalization and counter-radicalization research. Sociology of Crime, Law and Deviance, 25, 209-224.

Stemmeler, S. (2020). Kognitives und affektives Lernen durch eine schulbasierte Präventionsmaßnahme eines Aussteigers aus der rechtsextremen Szene - Eine lerntheoretische Analyse. In M. Walsh \& A. Gansewig (Hrsg.), Frühere Extremisten in der schulischen Präventionsarbeit. Perspektiven aus Wissenschaft und Praxis. Berichte des Nationalen Zentrums für Kriminalprävention, Nr. 1/2020. (S. 35-44). Bonn: Nationales Zentrum für Kriminalprävention. 
Szukala, A., \& Davies, I. (2020). The interconnections between research and impact in social sciences education. Journal of Social Science Education, 19, 1-7.

Walsh, M. (2020). Evidenzorientierung in der deutschen Kriminalprävention und -politik. Entwicklung und Überlegungen zum Stand der Dinge. Neue Kriminalpolitik, 1, 24-34.

Walsh, M., \& Gansewig, A. (2019). Evaluationsstudie einer schulbasierten Präventionsmaßnahme eines Aussteigers aus dem Bereich Rechtsextremismus. Abschlussbericht. Forschungsberichte des Nationalen Zentrums für Kriminalprävention, Nr. 2/2019. Bonn: Nationales Zentrum für Kriminalprävention.

Walsh, M., \& Gansewig, A. (2020). Überlegungen zum evidenzorientierten Handeln beim schulischen Einsatz ehemaliger Extremisten. In M. Walsh \& A. Gansewig (Hrsg.), Frühere Extremisten in der schulischen Präventionsarbeit. Perspektiven aus Wissenschaft und Praxis. Berichte des Nationalen Zentrums für Kriminalprävention, Nr. 1/2020. (S. 9-15). Bonn: Nationales Zentrum für Kriminalprävention. 